Research Paper

\title{
Isolation and determination of four potential antimicrobial components from Pseudomonas aeruginosa extracts
}

Ling-Qing $\mathrm{Xu}^{1}$, Jian-Wen Zeng ${ }^{2}$, Chong-He Jiang ${ }^{2}$, Huan Wang ${ }^{1}$, Yu-Zhen Li ${ }^{1}$, Wei-Hong Wen ${ }^{1}$, Jie-Hua Li ${ }^{1}$,



1. Department of Clinical Laboratory, The Sixth Affiliated Hospital of Guangzhou Medical University, Qingyuan People's Hospital, Guangdong, China;

2. Department of Urology, The Sixth Affiliated Hospital of Guangzhou Medical University, Qingyuan People's Hospital, Guangdong, China;

3. Department of Clinical Laboratory, Tongji Hospital, Tongji Medical College, Huazhong University of Science and Technology, Hubei, China;

4. Graduate Institute of Basic Medical Science, China Medical University, Taichung, Taiwan;

5. Graduate Institute of Chinese Medical Science, China Medical University, Taichung, Taiwan;

6. Department of Health and Nutrition Biotechnology, Asia University, Taichung, Taiwan.

* These authors have contributed equally to this paper

$\bowtie$ Corresponding author: Chih-Yang Huang, cyhuang@mail.cmu.edu.tw.

(c) Ivyspring International Publisher. This is an open access article distributed under the terms of the Creative Commons Attribution (CC BY-NC) license (https://creativecommons.org/licenses/by-nc/4.0/). See http://ivyspring.com/terms for full terms and conditions.

Received: 2016.12.23; Accepted: 2017.10.11; Published: 2017.11.02

\begin{abstract}
Background: Pseudomonas aeruginosa can cause disease and also can be isolated from the skin of healthy people. Additionally, it exhibits certain antimicrobial effects against other microorganisms.

Methods: We collected 60 strains of $P$. aeruginosa and screened their antimicrobial effects against Staphylococcus aureus (ATCC 25923) using the filter paper-disk method, the cross-streaking method and the co-culture method and then evaluated the antimicrobial activity of the chloroform-isolated S. aureus extracts against methicillin-resistant S. aureus (MRSA, Gram-positive cocci), vancomycin intermediate-resistant S. aureus (VISA, Gram-positive cocci), Corynebacterium spp. (CS, Gram-positive bacilli), Acinetobacter baumannii (AB, Gram-negative bacilli), Moraxella catarrhalis (MC, Gram-negative diplococcus), Candida albicans (CA, fungi), Candida tropicalis (CT, fungi), Candida glabrata (CG, fungi) and Candida parapsilosis (CP, fungi).

Results: The PA06 and PA46 strains have strong antimicrobial effects. High-performance liquid chromatography (HPLC) analysis revealed that the major components of PA06 and PA46 that exhibit antimicrobial activity are functionally similar to phenazine-1-carboxylic acid (PCA) and pyocyanin. Preparative HPLC was performed to separate and isolate the 4 major potential antimicrobial components: PA06ER10, PA06ER16, PA06ER23 and PA06ER31. Further, the molecular masses of PA06ER 10 (260.1), PA06ER16 (274.1), PA06ER23 (286.1) and PA06ER31 (318.2) were determined by electrospray ionization (ESI) mass spectrometry.

Conclusion: $P$. aeruginosa can produce small molecules with potential antimicrobial activities against MRSA, VISA, CS, MC, CA, CT, CG and CP but not against AB.
\end{abstract}

Key words: Pseudomonas aeruginosa; Staphylococcus aureus; antimicrobial effects; PCA; pyocyanin.

\section{Introduction}

Microbial active metabolites are the sources of most antimicrobials used for the treatment of various infections. Since the discovery of penicillin in 1928, studies on bacteria and fungi have revealed that microorganisms are rich in bioactive substances [1]. Since penicillin, many other drugs have been discovered from microorganisms [2]. Currently, many pathogens implicated in infectious disease are rapidly developing resistance against available antimicrobials, making the treatment of infectious diseases difficult. Therefore, more effective antimicrobials must be discovered $[3,4]$. 
Pseudomonas aeruginosa is a common Gram-negative bacterium that has been shown to produce secondary metabolites, phytotoxins, slime and antifungals, which confer obvious selective advantages to these organisms in their environment [5-8]. P. aeruginosa was further reported to produces a variety of redox-active phenazine compounds, including pyocyanin, phenazine-1-carboxylic acid (PCA), 1-hydroxyphenazine and phenazine-1carboxamide [9-10].

Although $P$. aeruginosa can cause disease, it cannot be isolated from the skin of healthy people. In some cases, in the absence of treatment, $P$. aeruginosa can mature and develop a larger population in the host body, indicating that it has the potential for antimicrobial production. In some clinical antimicrobial treatments, $P$. aeruginosa has shown the ability to develop resistant strains, and $P$. aeruginosa analogues can kill other clinical antimicrobial resistant strains.

In the present study, we investigated the antifungal and antimicrobial properties of $P$. aeruginosa metabolites against other microorganisms. We found that $P$. aeruginosa can produce small molecule antimicrobials other than PCA and pyocyanin.

\section{Materials and Methods}

\section{Strains}

Sixty non-repetitive strains of $P$. aeruginosa were obtained from various specimens from the in-patient department in our hospital between 2013 and 2015 and were identified by the Gram-stain assay, oxidase test and Vitek-2 automated microbial identification system (Merieux, France) or API-20NE (Merieux, France). The standard bacteria, including Escherichia coli (ATCC 25922), Klebsiella pneumoniae (ATCC 700603), P. aeruginosa (ATCC 27853), Bacillus thuringiensis (ATCC13838), Staphylococcus aureus (ATCC 25923), Proteus vulgaris (ATCC 6380), and Candida albicans (CA, ATCC90028), were used as calibration controls in the lab. Bacteria were grown on nutrient agar and, when appropriate, on $7 \% \mathrm{v} / \mathrm{v}$ blood agar or Sabouraud dextrose agar.

\section{Screening the antimicrobial effects of $P$. aeruginosa strains}

The 60 strains were screened for antibacterial effects against $S$. aureus ATCC 25923 using the disk diffusion method. A total volume of $400 \mu$ l of $1.5 \times 10^{8}$ cells/ml of $S$. aureus ATCC 25923 (for other microorganisms, we used the same method) was spread on Mueller-Hinton agar $(\mathrm{MH})$ plates (fungi were spread on Sabouraud dextrose agar oxoid plates) with a glass spreader. Then, sterile filter paper disks were placed on the plates. Each $P$. aeruginosa strain was grown overnight at $37^{\circ} \mathrm{C}$ in Luria-Bertani (LB) medium, and $3 \mu \mathrm{l}$ of an $8 \times 10^{9}-\mathrm{CFU} / \mathrm{ml} P$. aeruginosa culture was spotted on each filter disk, followed by incubation at $30^{\circ} \mathrm{C}$ for up to $48 \mathrm{~h}$. The PA06 and PA46 strains produced the largest zones and were, therefore, selected for subsequent studies. PA01 and PA22, which showed no effect, were also chosen for further study.

\section{Cross-streaking assay}

According to the method described by Kerr et al. $[5,11]$, an agar plate was seeded with a 4-h LB culture of a strain of $P$. aeruginosa, which was applied with a sterile cotton swab in a 1-cm-wide streak. After incubation at $37^{\circ} \mathrm{C}$ for $24 \mathrm{~h}$, the bacterial growth was removed from the plate with a microscope slide, and the residual microorganisms were killed by exposure to chloroform vapor for $30 \mathrm{~min}$. LB cultures of other microorganisms were diluted in fresh broth and streaked with a loop at right angles to the line of the original inoculum. The plates were incubated at $37^{\circ} \mathrm{C}$ for $18 \mathrm{~h}$ and examined for inhibition of the indicator strain in the area that had supported the growth of the producer strain.

\section{Co-culture with $P$. aeruginosa}

Each sterile Eppendorf tube was filled with $1 \mathrm{ml}$ of LB medium. Then, $50 \mu \mathrm{l}$ of an $8 \times 10^{9}-\mathrm{CFU} / \mathrm{ml} P$. aeruginosa culture was added into the tube, followed by $50 \mu \mathrm{l}$ of the indicator microorganism and mixing. The mixture was shaken at $250 \mathrm{rpm}$ at $30^{\circ} \mathrm{C}$ for $48 \mathrm{~h}$. Then, $5 \mu \mathrm{l}$ of the mixture was utilized for the Gram-stain assay. After air-drying, the samples were observed via microscopy and imaged at 1000x.

\section{P. aeruginosa extract preparation}

Each of the four $P$. aeruginosa strains (PA01, PA06, PA22 and PA46) was inoculated into $2 \mathrm{~L}$ of nutrient broth and incubated at $37^{\circ} \mathrm{C}$ for 7 days. The culture was then centrifuged at $10000 \mathrm{rpm}$ for $15 \mathrm{~min}$. Subsequently, the supernatant was filtered, extracted with chloroform and dried at room temperature $\left(25^{\circ} \mathrm{C}\right)$. The obtained extracts were weighed and kept in a desiccator for later use.

\section{Isolation of potential components from the extracts}

Extracts were dissolved in $1 \mathrm{ml}$ of methanol and then filtered through $0.22 \mu \mathrm{m}$ hydrophobic membranes. Analytical high-performance liquid chromatography (HPLC) was performed on an instrument (LC-20AD, SHIMADZU, Japan) equipped with a detector (UV-Vis 190-600 nm, SPD-20A) and integrated CSW 32 software, using an Apollo C18 
column $(250 \mathrm{~mm} \times 4.6 \mathrm{~mm}, 5 \mu \mathrm{m}$ integrated precolumn). The mobile phase consisted of a $30 \mathrm{~min}$, $40 \%$ to $60 \%$ linear gradient of acetonitrile in water. The flow rate was $1.0 \mathrm{ml} / \mathrm{min}$, and the chromatograms were monitored at $256 \mathrm{~nm}$. The spectra were analyzed with a 990 photodiode array detector (Waters). Preparative HPLC was performed on a KNAUER Smartline (Germany) system equipped with a detector (UV-Vis 190-600 nm, SPD-20A) and integrated CSW32 software, using a Eurospher C18 column $(10 \mathrm{~mm} \times 250 \mathrm{~mm}, 5 \mu \mathrm{m}$ integrated precolumn), under the same conditions used for analytical HPLC, except that the flow rate was 4.0 $\mathrm{ml} / \mathrm{min}$. The eluate of every peak was collected in a clean tube, and concentrated in a desiccator. Using $S$. aureus (ATCC 25923) as an indicator bacterium, the inhibitory effects of the eluates were observed by the agar-well diffusion method.

\section{Electrospray lonization Mass Spectrometry (ESI-MS)}

ESI-MS was performed in positive ion fast atom bombardment mode on a QTRAP 4500 MS (AB SCIEX, USA) and in gas chromatograph-MS using a $15 \mathrm{~mm}$ DB5 capillary column.

\section{Results}

\section{Antimicrobial effects of $P$. aeruginosa strains against other microorganisms}

A total of 60 strains of $P$. aeruginosa were screened. Overall, 35 strains of the $P$. aeruginosa isolates exhibited a strong inhibitory effect against $S$. aureus (ATCC 25923), while the other $P$. aeruginosa strains had no effect (Figure 1A). In particular, $P$. aeruginosa PA06 and PA46 showed the largest inhibitory zone effects, whereas PA01 and PA22 had no effects. Further studies revealed that PA06 and PA46 exerted significant inhibitory effects on methicillin-resistant S. aureus (MRSA, Gram-positive cocci), vancomycin intermediate-resistant S. aureus (VISA, Gram-positive cocci), Corynebacterium spp. (CS, Gram-positive bacilli), Acinetobacter baumannii (AB, Gram-negative bacilli), Moraxella catarrhalis (MC, Gram-negative diplococcus) and fungi such as CA, Candida tropicalis (CT), Candida glabrata (CG), and Candida parapsilosis (CP). However, PA01 and PA22 had no inhibitory effect on Gram-negative bacilli, such as $A B$ (Figure 1B).

(A)

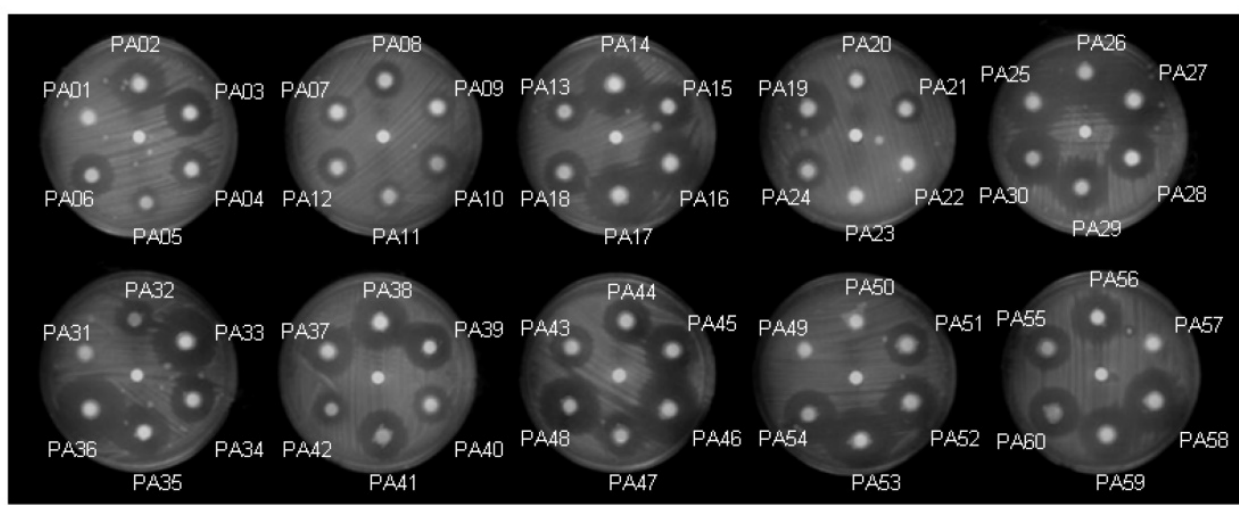

(B)

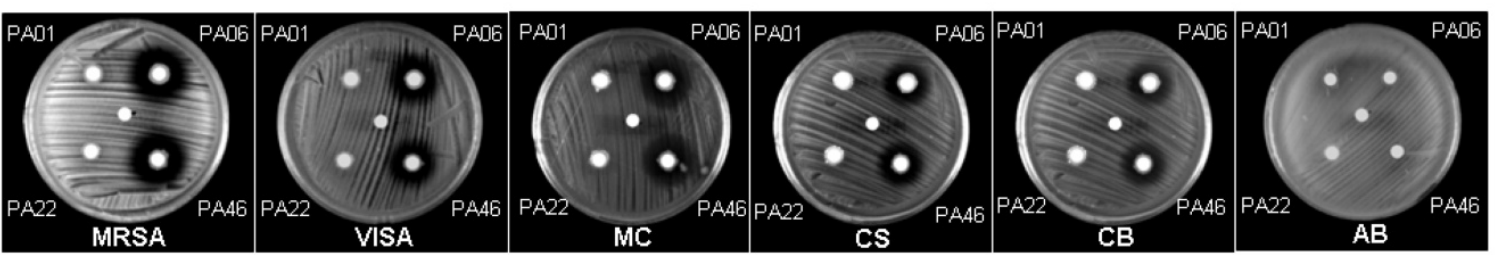

(C)

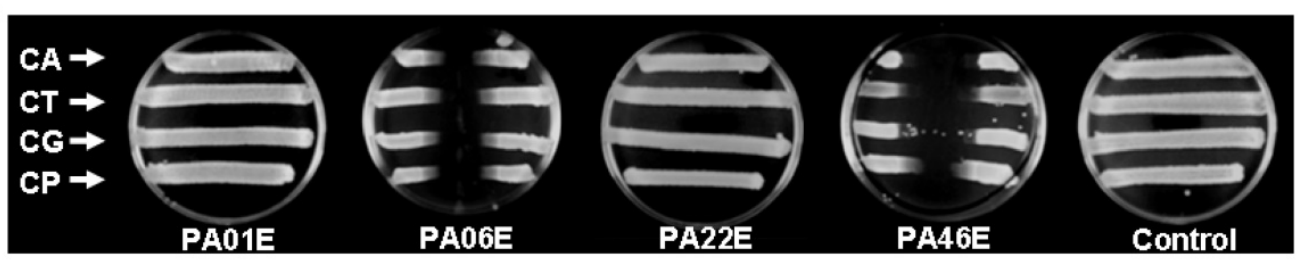

Figure 1. P. aeruginosa antimicrobial array. (A) 60 strains of $P$. aeruginosa were co-cultured with S. aureus (ATCC25923) for 24 h, and 35 strains of $P$. aeruginosa strains showed antimicrobial effects against $S$. aureus. Two strains of $P$. aeruginosa (PA06 and PA46) exhibited the strongest inhibitory effects (area) against $S$. aureus growth. (The center dot of each plate is sterile water.) (B) PA01, PA06, PA22 and PA46 were further evaluated, and their antimicrobial effects on MRSA (Gram-positive cocci), VISA (Gram-positive cocci), MC and CS were assessed. PA06 and PA46 showed no antimicrobial effects on AB. (C) In cross-streaking tests, the parallel inoculums were CA (ATCC 90028), CT, CG and $\mathrm{CP}$ in all plates. The center vertical coating of each plate was a P. aeruginosa strain extract (PA01E, PA06E, PA22E, or PA46E) or the control (chloroform only). Both the PA06 and PA46 strains presented broad antimicrobial effects, unlike the PA01 and PA22 strains. 


\section{Isolation and characterization of extracts}

Analytical HPLC showed that both the PA01 and PA22 P. aeruginosa strains had similar patterns, while the PA06 and PA46 P. aeruginosa strains were similar to each other (Figure 3) However, they were different from the PCA and pyocyanin reference standards. As a result, we subjected an extract of PA06 P. aeruginosa strain to preparative HPLC for further study. The preparative HPLC revealed that the PA06 extract (PA06E) contained six major components: PA06ER07, PA06ER10, PA06ER13, PA06ER16, PA06ER23 and PA06ER31 in Figure 4A.
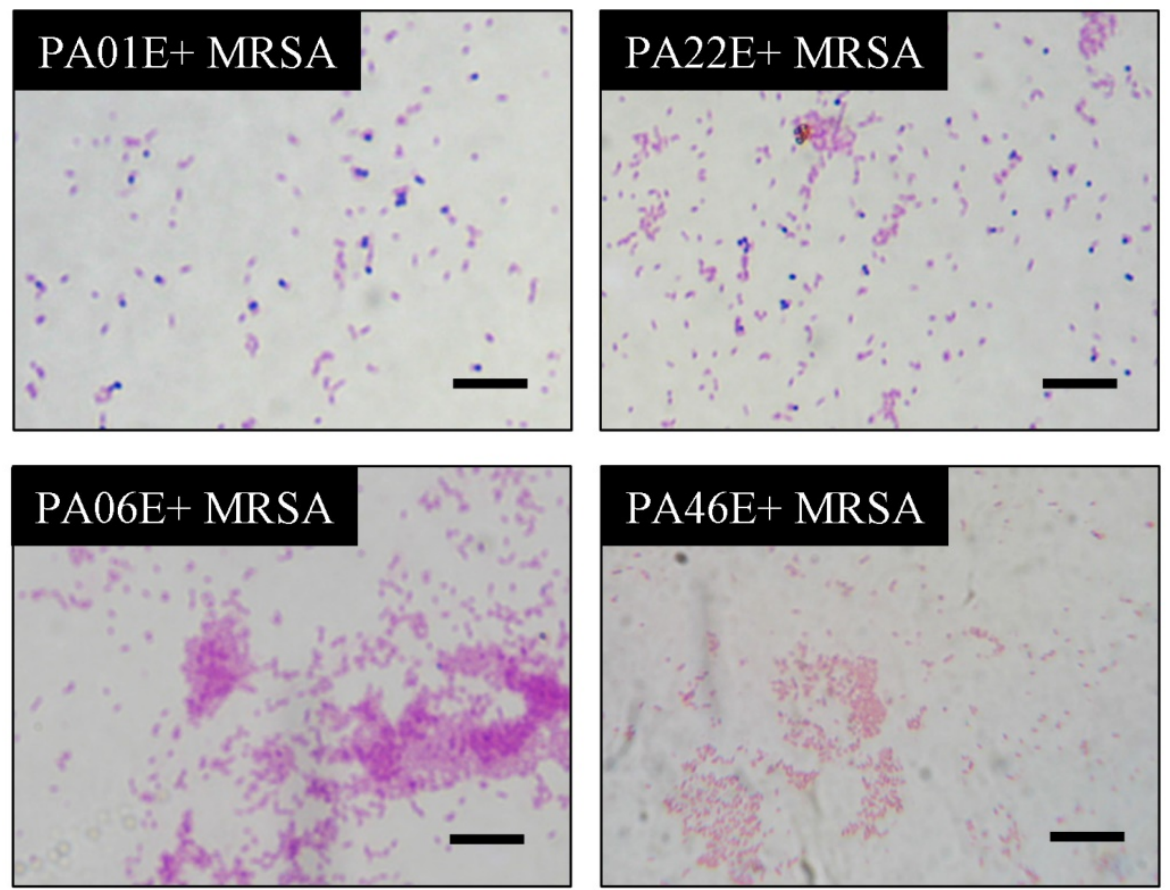

Figure 2. Antimicrobial effects of selected $P$. aeruginosa strains. The PA01, PA06, PA22 and PA46 strains of $P$. aeruginosa were co-cultured with MRSA in LB medium for $24 \mathrm{~h}$, and then, Gram-positive cocci were stained blue and Gram-negative bacilli pink. In the slices of MRSA co-cultured with PA06 and PA46, only Gram-negative bacilli can be detected. In the slice of MRSA co-cultured with PA01 and PA22, both Gram-negative bacilli and Gram-positive cocci can be detected (scale bar length is $1 \mu \mathrm{m}$ ).

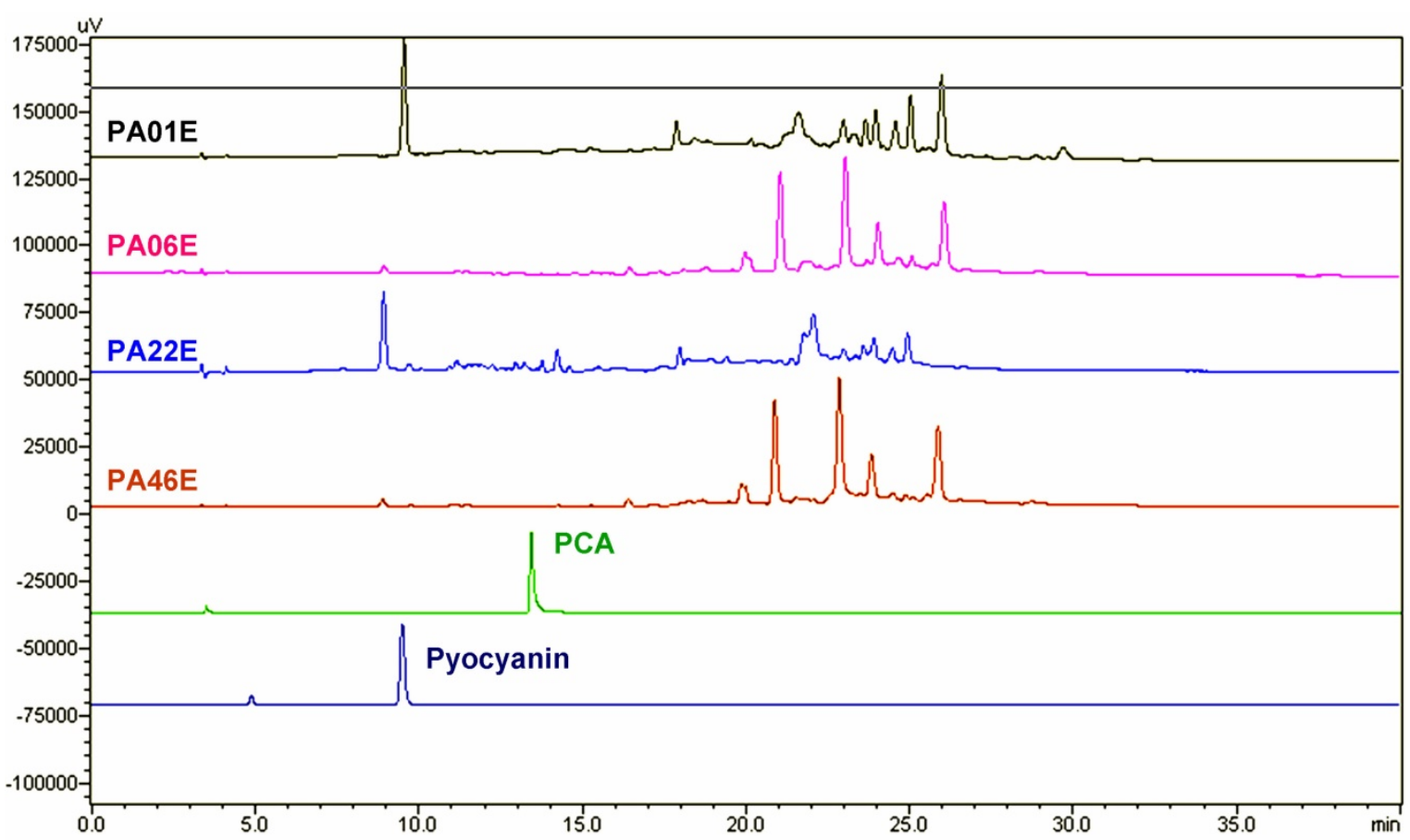

Figure 3. HPLC analysis of $P$. aeruginosa extracts. The PA01, PA06, PA22 and PA46 strains of $P$. aeruginos $a$ were collected and extracted. The extracts of each strain from $P$. aeruginosa were further analyzed and compared with those of PCA (Retention time, $\mathrm{Rt}=13.247$ seconds) and pyocyanin $(\mathrm{Rt}=9.336$ second). 
(A)

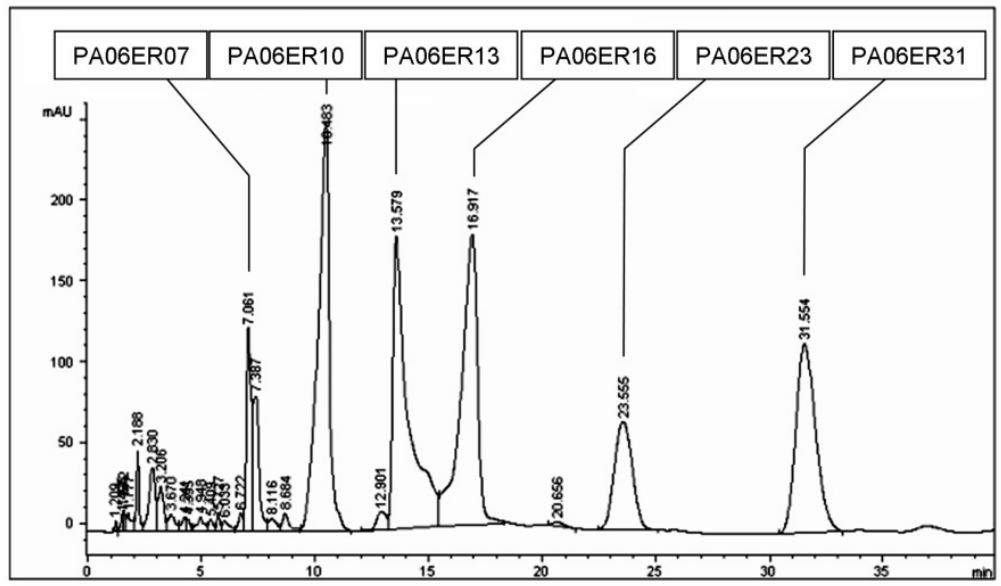

(B)

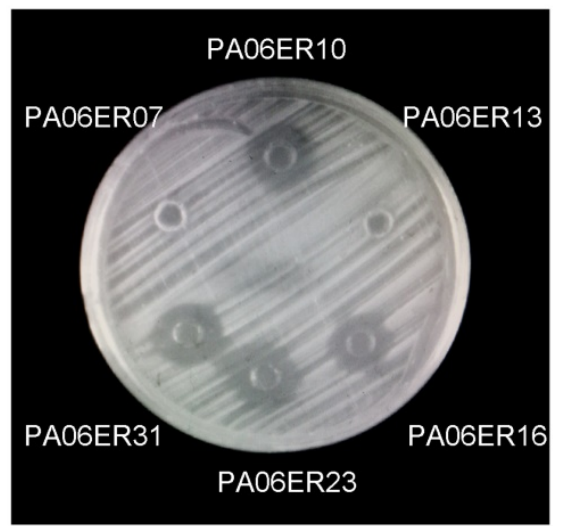

Figure 4. HPLC analysis of the major components of PA06 extracts. (A) The components of the PA06 extract were analyzed and isolated by preparative HPLC. The 6 major components of PA06 were identified and labeled as PA06ER07 (Rt $=7.061$ seconds), PA06ER $10(\mathrm{Rt}=10.483$ seconds), PA06ER 13 (Rt $=13.579$ seconds), PA06ER16 (Rt = 16.917 seconds), PA06ER23 (Rt $=23.555$ seconds), and PA06ER31 (Rt $=31.554$ seconds). (B) These 6 major components were further co-cultured with S. aureus (ATCC25923). Only PA06E R10, PA06ER16, PA06ER23 and PA06ER31 presented antimicrobial effects against S. aureus (ATCC 25923).

Every eluate of the major components was collected and concentrated to $0.5 \mathrm{ml}$, and then, their antimicrobial activities against $S$. aureus were further tested. PA06ER07 and PA06ER13 exhibited no antimicrobial effects against $S$. aureus, but PA06ER10, PA06ER16, PA06ER23 and PA06ER31 displayed strong inhibitory effects on $S$. aureus (Figure 4B). ESI-MS was applied for the molecular mass determination of the potential antimicrobial components. The MS spectra demonstrated that the molecular weights of PA06ER10, PA06ER16, PA06ER23, and PA06ER31 were $260.1 \mathrm{~g} / \mathrm{mol}, 274.1$ $\mathrm{g} / \mathrm{mol}, 286.1 \mathrm{~g} / \mathrm{mol}$ and $318.2 \mathrm{~g} / \mathrm{mol}$, respectively (Figure $5 \mathrm{ABCD}$ ).

\section{Discussion}

Some reports have shown interactions between $P$. aeruginosa and microorganisms in the human body. Hughes et al. [12] demonstrated that in cystic fibrosis (CF) patients with $P$. aeruginosa infection, only $10 \%$ had positive CA skin tests, compared with $30 \%$ in those free of $P$. aeruginosa, suggesting that the antifungal substance produced by $P$. aeruginosa could prevent Candida infections. Other reports have suggested that $P$. aeruginosa inhibits the growth of Cryptococcus species $[13,14]$. However, no report has addressed the isolation of Cryptococcus species from CF patients. Considering that both Cryptococcus and $P$. aeruginosa are common lung pathogens, the lack of co-colonization could be the result of an antifungal effect of $P$. aeruginosa on the growth of Cryptococcus neoformans.

Grillot et al. investigated the interactions between $P$. aeruginosa and yeast after incubation with a series of pure and mixed cultures [14]. They demonstrated that the growth of all tested isolates was inhibited by this bacterium in blood culture medium and bacterial culture filtrates. Hogan and Kolter described a pathogenic interaction between $P$. aeruginosa and CA, observing that $P$. aeruginosa forms a dense biofilm on CA filaments and kills the fungus [15]. Several $P$. aeruginosa virulence factors (Type IV pili, phospholipase $\mathrm{C}$ and phenazines) that are important in disease are involved in the killing of CA filaments. Both pyocyanin and pseudomonas quinolone signal (PQS) intensively accumulate in the lung mucus of CF patients, and these antifungal molecules may be important in the prevention of pulmonary cryptococcosis in CF patients $[16,17]$.

Most antimicrobial compounds for clinical use have been isolated from microorganisms. Additionally, several previous reports have identified antimicrobial factors from $P$. aentginosas [18-20]. In this work, we isolated several potential antibiotic compounds from $P$. aentginosas strains (Figure 1). These potential antibiotic compounds exhibited significant growth inhibition against $S$. aureus, especially MRSA, which is methicillin-resistant Gram-positive cocci (Figure 2). These results suggested that the components of PA06E and PA46E are not methicillin. The activities described here against $S$. aureus are similar to those in Machan's report from 1995, although they did not reveal more details about the substance [21]. Following that report, some antimicrobials with phenazine-like structures, including PCA and pyocyanin, were discovered and isolated from $P$. aeruginosa $[22,23]$. 
(A)



(B)



(C)

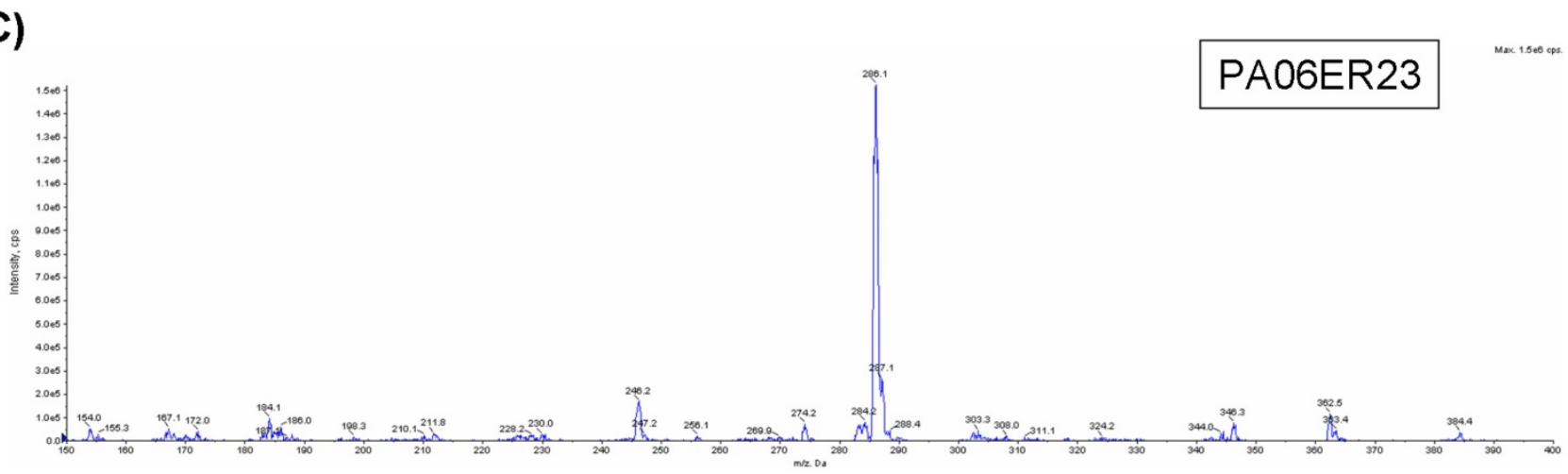

(D)

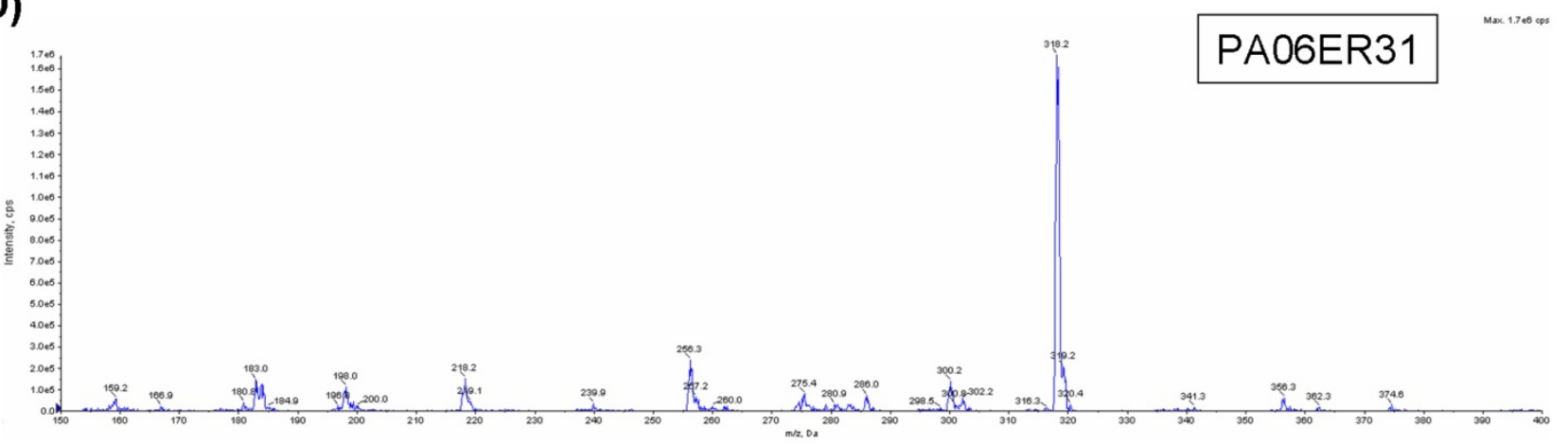

Figure 5. ESI-MS analysis of the components with the potential antimicrobial activity. (A) The molecular mass of PA06ER 10 is $260.1 \mathrm{~g} / \mathrm{mol}$. (B) The molecular mass of PA06ER 16 is $274.1 \mathrm{~g} / \mathrm{mol}$. (C) The molecular mass of PA06ER23 is $286.1 \mathrm{~g} / \mathrm{mol}$. (D) The molecular mass of PA06ER31 is $318.2 \mathrm{~g} / \mathrm{mol}$.

In the present study, we investigated the abilities of the extracts from PA06 and PA46 strains to inhibit the growth of other microorganisms. Our results demonstrated that these two strain extracts have strong inhibitory effects against Gram-positive cocci, Gram-positive bacilli, fungi and Gram-negative 
diplococcus but no inhibitory effects on Gram-negative bacilli. The inhibitory rates of these two strains against Gram-positive cocci (including MRSA and VISA) were $100 \%$. Some P. aeruginosa strains had strong antifungal abilities, but others displayed only partial or no abilities.

The HPLC analysis provided strong evidence suggesting that the major antimicrobial components of PA06E and PA46E do not include PCA and pyocyanin (Figure 3). Furthermore, the ESI-MS analysis of the potential antimicrobial components of PA06E proved that the major compounds with antimicrobial activity were PA06ER10, PA06ER16, PA06ER23 and PA06ER31 and not PCA and pyocyanin (Figure 4). According to the antimicrobial spectrum analysis, PCA, pyocyanin, cephalosporins, phenethicillin, oritavancin, vancomycin and similar compounds present antimicrobial activities against Gram-positive rather than Gram-negative bacteria. In contrast, only compounds with pyocyanin-like structures, such as PCA and pyocyanin, exhibit antimicrobial activities against Gram-positive bacteria and fungi [24-30]. Therefore, PA06ER10, PA06ER16, PA06ER23 and PA06ER31 might be phenazine analogs because of their similar functions and heavier molecular weights (Figure 4). However, the exact structures of these compounds require further analysis and experimental support.

In summary, $P$. aeruginosa has potent antimicrobial activities. The underlying mechanism seems to be the production of small molecular substances that are distinct from PCA or pyocyanin. These potential antimicrobial components have strong inhibitory effects against Gram-positive cocci and Gram-negative diplococcus but not against Gram-negative bacilli. These findings will be meaningful for the treatment and prevention of various infections in the clinic, especially MRSA, VISA, and fungal infections.

\section{Acknowledgements}

This work was supported by the Infectious Diseases Control Project of the Ministry of Health of China (No. 2012ZX10004207-004) and the Science and Technology Project of Qingyuan City (No. 2014A005).

\section{Competing Interests}

The authors have declared that no competing interest exists.

\section{References}

1. Fenical W. Chemical studies of marine bacteria: developing a new resource. Chem Rev. 1993, 93: 1673-83.

2. Tawiah AA, Gbedema SY, Adu F, et al. Antibiotic producing microorganisms from River Wiwi, Lake Bosomtwe and the Gulf of Guinea at Doakor Sea Beach, Ghana. BMC Microbiology. 2012, 12: 234.
3. Singer RS, Finch $\mathrm{R}$, Wegener $\mathrm{HC}$, et al. Antibiotic resistance the interplay between antibiotic use in animals and human being. Lancet Infect Dis. 2003, 3: 47-51.

4. Bhavnali SM, Ballow $\mathrm{CH}$. New agent for Gram-positive bacteria. Curr Op Microbiol. 2000, 3: 528-34.

5. Kerr JR. Suppression of fungal growth exhibited by Pseudomonasaeruginosa. J Clin Microbiol. 1994, 2: 525-7.

6. Hockey LJ, Fujita NK, Gibson TR, et al. Detection of fungemia obscured by concomitant bacteremia: in vitro and in vivo studies. J Clin Microbiol. 1982. 6: 1080-5.

7. Kandela SA, al-Shibib AS, al-Khayat BH. A study of purified pyorubin produced by local Pseudomonas aeruginosa. Acta Microbiol Pol. 1997, 1: 37-43.

8. Kerr JR, Taylor GW, Rutman A, et al. Pseudomonas aeruginosa pyocyanin and 1-hydroxyphenazine inhibit fungal growth. J Clin Pathol. 1999, 5: 385-7.

9. Mavrodi DV, Bonsall RF, Delaney SM, et al. Functional analysis of genes for biosynthesis of pyocyanin and phenazine-1-carboxamide from Pseudomonas aeruginosa PAO1. J Bacteriol. 2001, 183: 6454-65.

10. Allen L, Dockrell DH, Pattery T et al. Pyocyanin production by Pseudomonas aeruginosa induces neutrophil apoptosis and impairs neutrophil-mediated host defenses in vivo. J Immunol. 2005, 174: 3643-9.

11. Nostro A, Germanò $M, D^{\prime}$ Angelo $V$, et al. Extraction methods and bioautography for evaluation of medicinal plant antimicrobial activity. Lett Appl Microbiol. 2000, 30: 379-384.

12. Hughes WT, Kim HK. Mycoflora in cystic fibrosis: some ecologic aspects of Pseudomonas aeruginosa and Candida albicans. Mycopathol Mycol Appl. 1973, 50: 261-269.

13. Grillot R, Portmann-Coffin V, Ambroise-Thomas P. Growth inhibition of pathogenic yeasts by Pseudomonasaeruginosa in vitro: clinical implications in blood cultures. Mycoses. 1994, 37: 343-347.

14. Hogan DA, Kolter R. Pseudomonas-Candida interactions: an ecological role for virulence factors. Science. 2002, 296: 2229-2232.

15. Grillot R, Portmann-Coffin V, Ambroise-Thomas P. Growth inhibition of pathogenic yeasts by Pseudomonasaeruginosa in vitro: clinical implications in blood cultures. Mycoses. 1994, 37: 343-347.

16. Taylor GW, Machan ZA, Mehmet S, et al. Rapid identification of 4-hydroxy-2-alkylquinolines produced by Pseudomonas aeruginosa using gas chromatography- electron-capture mass spectrometry. J Chromatogr B Biomed Appl. 1995, 664: 458-462.

17. Caldwell CC, Chen Y, Goetzmann HS, et al. Pseudomonas aeruginosa exotoxin pyocyanin causes cystic fibrosis airway pathogenesis. Am J Pathol. 2009, 175: 2473-2488

18. Young G. Pigment production and antibiotic activity in cultures of Pseudomonas aeruginosa. J Bacterioll. 1947, 54: 109-117.

19. Fuller AT, Banks GT, Mellows G, et al. Pseudomonic acid: an antibiotic produced by Pseudomonasfluorescens. Nature. 1971, 234: 416-417.

20. Hays EE, Wells IC, Katzman PA, et al. Antibiotic substances produced by Pseudomonas aeruginosa. J Biol Chem. 1945, 159: 725-750.

21. Taylor GW, Machan ZA, Mehmet S, et al. Rapid identification of 4-hydroxy-2-alkylquinolines produced by Pseudomonas aeruginosa using gas chromatography- electron-capture mass spectrometry. J Chromatogr B Biomed Appl. 1995, 664: 458-642.

22. Zhang L, Tian X, Kuang S, et al. Antagonistic Activity and Mode of Action of Phenazine-1-Carboxylic Acid, Produced by Marine Bacterium Pseudomonas aeruginosa PA31x, Against Vibrio anguillarum In vitro and in a Zebrafish In vivo Model. Front Microbiol. 2017, 8: 289.

23. Polisetti S, Baig NF, Morales-Soto N, et al. Spatial Mapping of Pyocyanin in Pseudomonas Aeruginosa Bacterial Communities Using Surface Enhanced Raman Scattering. Appl Spectrosc. 2017, 71: 215-223.

24. Baron SS, Rowe JJ. Antibiotic action of pyocyanin. Antimicrob Agents Chemother. 1981, 20: 814-820.

25. Russel AD. Interaction of a new cephalosporin, 7-cyanacetamidocephalosporanic acid, with some gram-negative and gram-positive beta-lactamase-producing bacteria. Antimicrob Agents Chemother. 1972, 2: 255-260.

26. Sader HS, Jones RN, Stilwell MG, Flamm RK. Ceftaroline activity tested against uncommonly isolated Gram-positive pathogens: report from the SENTRY Antimicrobial Surveillance Program (2008-2011). Int J Antimicrob Agents. 2014, 43: 284-286.

27. Karlowsky JA, Walkty AJ, Baxter MR, et al. In vitro activity of Oritavancin against gram-positive pathogens isolated in Canadian hospital laboratories from 2011 to 2015. Diagn Microbiol Infect Dis. 2017, 87: 349-356.

28. Wei WX, Qin $\mathrm{XL}$, Cheng DH, et al. Retrospective analysis of vancomycin treatment outcomes in Chinese paediatric patients with suspected Gram-positive infection. J Clin Pharm Ther. 2016, 41: 650-656.

29. Relhan N, Albini TA, Pathengay A, et al. Endophthalmitis caused by Gram-positive organisms with reduced vancomycin susceptibility: literature review and options for treatment. Br J Ophthalmol. 2016, 100: 446-452.

30. Kerr JR, Taylor GW, Rutman A, et al. Pseudomonas aeruginosa pyocyanin and 1-hydroxyphenazine inhibit fungal growth. J Clin Pathol. 1999, 52: 385-387. 